\title{
Estudio de los Beneficios Percibidos por los Usuarios a través de su Experiencia en las Redes Sociales Hoteleras
}

\author{
Miguel Á. Sánchez-Jiménez ${ }^{(1)}$, María T. Fernández-Alles ${ }^{(1)}$ y Juan J. Mier-Terán-Franco ${ }^{(1)}$ \\ (1) Dpto. de Marketing y Comunicación, Universidad de Cádiz. INDESS (Instituto Universitario para el \\ Desarrollo Social Sostenible), Calle Ancha, 16, 11001 Cádiz, España. (e-mail: \\ miguelangel.sanchez@uca.es; teresa.alles@uca.es; juanjose.mier-teran@uca.es)
}

Recibido Jun. 20, 2018; Aceptado Ago. 21, 2018; Versión final Sep. 12, 2018, Publicado Feb. 2019

\begin{abstract}
Resumen
En esta investigación se pretende estudiar los beneficios que proporcionan las redes sociales de los hoteles a los usuarios. Las actividades de innovación están muy presentes en el sector turístico, la aparición de los medios sociales, que facilitan la creación, edición e intercambio de contenido, permiten a las empresas relacionarse de forma más directa e interactiva con los usuarios. Se ha realizado un análisis cuantitativo mediante un cuestionario dirigido a los seguidores de las redes sociales de hoteles o cadenas de hoteles. De esta manera se han propuesto 4 hipótesis basadas en el estudio de las relaciones entre la experiencia del usuario en las redes sociales de los hoteles y los 4 beneficios identificados (beneficio funcional, beneficio socio-psicológico, beneficio hedónico y beneficio monetario). Se pudo verificar así que una mejor experiencia en la red social del hotel provoca un aumento de los beneficios percibidos por el usuario.
\end{abstract}

Palabras clave: redes sociales; sector hotelero; usuarios; experiencia; beneficios

\section{Study of the Benefits Perceived by the Users Through their Experience in the Hotel Social Networks}

\begin{abstract}
This research aims to study the benefits provided by social networks of hotels to users. Innovation activities are very present in the tourism sector, the emergence of social media, which facilitate the creation, editing and exchange of content, allow companies to interact more directly and interactively with users. Thus, a quantitative analysis has been conducted through a questionnaire aimed at followers of social networks of hotels or hotel chains. In this way 4 hypotheses have been proposed based on the study of the relationships between the user experience in the social networks of the hotels and the 4 identified benefits: functional benefit, sociopsychological benefit, hedonic benefit and monetary benefit. It was possible to verify that better experience in the hotel's social network causes an increase in the benefits perceived by the user.
\end{abstract}




\section{INTRODUCCIÓN}

Las nuevas tecnologías de la comunicación (TIC) contribuyen a la rápida obsolescencia de los conocimientos y a la emergencia de nuevos valores, provocando continuas transformaciones en las estructuras económicas, sociales y culturales, e incidiendo en casi todos los aspectos de la vida, destacando su incorporación en el ámbito empresarial, que ha permitido mejorar la productividad de las empresas (Barrera, 2017; Arévalo-Avecillas et al., 2017). Por lo tanto, su gran impacto en todos los ámbitos hace cada vez más difícil que se pueda actuar eficientemente prescindiendo de las TIC. Además, se considera que las TIC forman parte de la cultura actual y amplían las posibilidades de desarrollo social personal. Suele incluirse en el concepto TIC no solamente la informática y sus tecnologías asociadas, telemática y multimedia, sino también los medios de comunicación de todo tipo: los medios de comunicación social y los medios de comunicación interpersonales tradicionales con soporte tecnológico como el dispositivo móvil (Martelo et al., 2017; Jiménez-Pitre et al., 2017).

Debido a la disponibilidad omnipresente de las TIC, las redes sociales han cambiado radicalmente la forma de comunicación de las organizaciones, las comunidades y los usuarios individuales y se han convertido en un lugar natural de intercambio de información Brendzel-skowera y Lukasik (2016). Las redes sociales son utilizadas por organizaciones e instituciones tanto como canal de comunicación como para construir interacciones sociales. En la actualidad, las empresas son conscientes que tienen que dirigirse al cliente de manera diferente, que deben gestionar la interacción y conversación con los consumidores, produciendo un feedback y una interactividad, ofreciendo una respuesta adecuada para satisfacer sus necesidades. En este sentido, internet se considera un entorno colaborativo, en el que las empresas y usuarios se encuentran e interactúan, y el consumidor participa, compartiendo experiencia u opiniones, influenciando directamente a otros usuarios (Martí, 2008). De esta manera, existen diversas herramientas, plataformas o medios sociales a través de las cuáles se puede producir la interacción y conversación entre comunidades de usuarios, estos medios sociales son entre otros: los blogs, los foros, las redes sociales generalistas (Facebook o Twitter), las redes sociales especializadas o temáticas (Tripadvisor), los servidores multimedia (Youtube), los fotologs (Instagram o Pinterest), en definitiva, cualquier medio a través de internet que permita la participación y la interactividad (Moreno, 2014; Zimmerman, 2015). Según Vila y Vila (2013), los medios sociales han ofrecido al consumidor la oportunidad de compartir opiniones, recomendaciones y experiencias a través de comentarios, imágenes o videos con otros usuarios, que pueden ser leídos y comentados entre ellos. Esto ha llevado a que muchas empresas turísticas se hayan lanzado a probarlo y tengan la oportunidad tanto de analizar la información como de comunicarse con el consumidor, dando paso a la promoción de ventas con el objetivo de crear un mensaje centrado en el cliente.

Las características propias del sector turístico, en general, y del sector hotelero, en particular, hacen que sea mayor su repercusión en los medios sociales. El sector turístico, como cualquier sector servicios, vende productos intangibles que se caracterizan por ser inseparables, perecederos y heterogéneos (Sirakayaa y Woodsideb, 2005), de ahí que el proceso de comunicación sea un elemento vital en la oferta (Sigala, 2007). Por esta razón, el uso de los medios sociales ha permitido nuevas posibilidades de comunicación y ha proporcionado un impacto sustancial en la demanda y la oferta turística (Martínez et al., 2013).

\section{Beneficios percibidos por los usuarios en las redes sociales}

A través de una comunidad de marca es posible fortalecer la lealtad de los usuarios y servir como un canal para la comunicación y el intercambio de conocimiento entre las marcas y su público objetivo (Bruhn et al., 2014). Así, muchos usuarios pueden utilizar estas redes sociales para mantenerse informado de lo que pasa alrededor del mundo eliminando barreras culturales, geográficas o físicas. Además, la investigación empírica ha demostrado que los consumidores que participan en comunidades de marcas tienden a tener una relación más profunda y más estrecha con la marca que aquellos que no lo son (Potgieter y Naidoo, 2017; Rosenthal y Brito, 2017). Varias teorías económicas y sociales han explicado por qué las personas utilizan las redes sociales (en este caso, los perfiles de redes sociales de las cadenas hoteleras): para obtener información; hacer compras; para comunicarse e interactuar con otros; para divertirse y disfrutar de la experiencia; para entablar nuevas relaciones; y para expresar sus opiniones y su identidad. Todas estas razones son contingentes a cada comunidad (Wang et al., 2002). De los estudios realizados sobre las investigaciones de Kang (2011), Leung et al. (2015), Vivek et al. (2012) y Naumann y Bowden (2015), y observadas las opiniones de expertos en redes sociales, se han identificado las principales variables objeto de estudio y propuesto varios modelos para contrastar las hipótesis que están relacionadas con el beneficio percibido por el usuario y el compromiso del consumidor.

Diversos investigadores han encontrado una teoría económica que explica la participación en comunidades online (Gu y Jarvenpaa, 2003). El valor del consumidor será creado si los consumidores perciben más beneficios ganados que costes. Entre los beneficios que perciben se encuentran las oportunidades de obtener 
fuentes de información y conocimiento, desarrollar relaciones interpersonales, etc. Los consumidores, por el contrario, sacrifican tiempo, atención y energía en recibir estos beneficios. Si los beneficios exceden a los sacrificios realizados, la comunidad aportará valor a sus miembros y el número de participantes crecerá (Butler, 2001). La participación de los miembros está relacionada significativamente con los beneficios que esperan recibir de la comunidad. Por lo tanto, aportando beneficios y creando valor a los miembros de las redes sociales se puede incentivar la participación e incrementar las compras realizadas.

Kang et al. (2007) hicieron hincapié en que tales beneficios se deberán dar constantemente. Si la comunidad no ofrece beneficios consistentes a los miembros de la comunidad, la actividad de la comunidad puede ponerse en riesgo. Cuando los miembros perciben beneficios que valen la pena, son más propensos a convertirse en participantes más activos (Morgan y Hunt, 1994). Distintos autores han mostrado interés por conocer los factores que motivan la participación y el compromiso de los usuarios de las redes sociales. Wang y Fesenmaier (2004) propusieron un modelo basado en las necesidades o beneficios que los usuarios buscan satisfacer en las comunidades virtuales. Según estos autores, la participación y el compromiso están impulsados por cuatro tipos de necesidades o beneficios: funcionales, sociales, psicológicos y hedónicos. Argumentan que para que las comunidades virtuales sean viables deben atender a una variedad de necesidades o beneficios de los miembros. En el estudio de Dholakia et al. (2004) identificaron factores específicos que motivan a los individuos a participar en comunidades virtuales basados en la acción intencional. Madupu (2006) afirmó que los motivos de Dholakia et al. (2004) pueden conciliarse y relacionarse con las categorías de beneficios propuestas por Wang y Fesenmaier (2004), proponiendo así un conjunto de antecedentes para la participación y compromiso en las comunidades virtuales. Además de estas cuatro variables, se tendrán en cuenta los beneficios monetarios que han sido valorados por otros estudios como el Gwinner et al. (1998) y Lee et al. (2008). Esta dimensión se relaciona con descuentos o eventos de la empresa ofrecidos a la comunidad "obtener descuentos o tratos especiales que la mayoría de consumidores no tienen", "obtener mejores precios que la mayoría de consumidores" y "recibir cupones gratis para una estancia en el hotel o de comida por hacerse miembro de la página de Facebook" (Tabla 1).

Tabla 1: Beneficios de la participación y el compromiso en las comunidades virtuales de una marca. (Adaptada de Kang (2011) y Wang y Fesenmaier (2004).

\begin{tabular}{|l|l|}
\hline Necesidades o beneficios & Factores motivacionales \\
\hline \multirow{3}{*}{ Funcionales } & Información/Utilidad \\
& Eficiencia \\
& Conveniencia \\
\hline \multirow{3}{*}{ Sociales } & Confianza \\
& Comunicación \\
& Relación \\
\hline \multirow{3}{*}{ Psicológicas } & Implicación \\
& Pertenencia \\
& Afiliación \\
\hline \multirow{3}{*}{ Hedónicas } & Identificación \\
& Entretenimiento \\
& Diversión \\
\hline \multirow{2}{*}{ Monetarias } & Placer \\
& Descuentos de precios \\
& Cupones gratis \\
\hline
\end{tabular}

En los estudios de Kang (2011) y Song y Yoo (2016) establecen que los beneficios sociales y psicológicos tienen una alta correlación entre ellos, por lo tanto, en ambos estudios, se combinaron ambas variables en una rebautizada como "beneficio socio-psicológico". Debido a que se ha demostrado alta correlación entre estas dos variables, en este estudio también se han combinado dando como resultado la variable "beneficio socio-psicológico" la cual ha sido objeto de estudio.

\section{La experiencia del usuario en las redes sociales}

En la investigación de Leung et al. (2015) se identificó que la experiencia del usuario en los medios sociales de marca es un factor muy importante en el social media marketing. Específicamente, un hotel debe hacer 
que sus páginas de medios sociales sean más interesantes, atractivas, informativas, interactivas y centradas en el consumidor para que los clientes puedan disfrutar de una buena experiencia. Además, la experiencia en social media por parte de los clientes en hoteles influencia su postura frente a la marca del hotel el cual es un factor importante que conduce a la intención de reservar. Por lo tanto, los hoteles deberían enfatizar el desarrollo de marca en sus páginas de redes sociales.

La realización de una gestión activa y atractiva para mejorar la experiencia del usuario por parte de las empresas ha sido tratada en varios estudios. Van der Lans et al. (2012) indican que las marcas deben ofrecer algo a los clientes que valga la pena, como, por ejemplo, unos contenidos atractivos, noticias, servicios exclusivos, etc. Dans (2011) defiende que la empresa debe escuchar activamente, participar en las conversaciones con un lenguaje próximo y cercano, ofrecer actualizaciones frecuentes con contenidos relevantes y de calidad, responder de manera rápida y transmitir transparencia y confianza. De esta manera, el objetivo de este estudio consiste en "contrastar si los usuarios perciben beneficios a través de una buena experiencia en la página de social media de los hoteles". Para ello se plantean 4 hipótesis que relacionan la experiencia del usuario en las redes sociales de los hoteles con los beneficios percibidos por los usuarios. Estas hipótesis se justifican en el siguiente apartado de revisión de la literatura.

\section{Hipótesis y modelo de investigación}

En la presente investigación se plantean las siguientes hipótesis que van a ser contrastadas empíricamente: H1) una mejor experiencia en la página de social media del hotel se asocia con un mayor beneficio funcional; H2) una mejor experiencia en la página de social media del hotel se asocia con un mayor beneficio sociopsicológico; H3) una mejor experiencia en la página de social media del hotel se asocia con un mayor beneficio hedónico; H4) una mejor experiencia en la página de social media del hotel se asocia con un mayor beneficio monetario.

Teniendo en cuenta lo anteriormente comentado es de interés estudiar si realmente las redes sociales de los hoteles van a proporcionar una serie de beneficios a los usuarios, teniendo en cuenta la experiencia de los usuarios en dichas páginas. A partir de las relaciones que se establecen entre estas variables y de los objetivos planteados se proponen los siguientes cuatro modelos de regresión lineal simple que se van a utilizar para contrastar las hipótesis 1, 2, 3 y 4 (Figura 1).

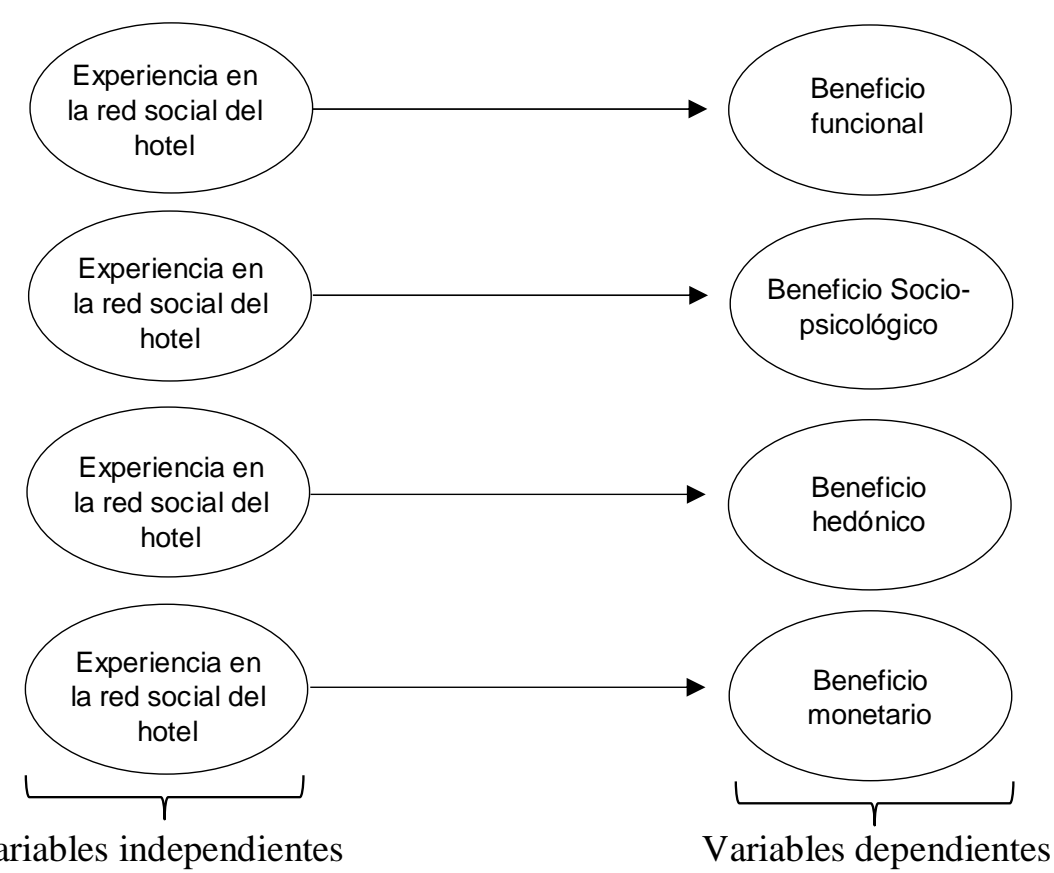

Fig. 1: Cuatro modelos de regresiones lineales simple. Relación entre la experiencia en la red social y los beneficios.

\section{METODOLOGIA}

Para alcanzar los objetivos se ha llevado a cabo un análisis cuantitativo fundamentado en los datos extraídos de un cuestionario dirigido a los usuarios de las redes sociales de los hoteles (Facebook y Twitter). De esta manera, se identifican las variables objeto de estudio y se proponen distintos modelos de regresión para 
estudiar las relaciones entre las variables y contrastar las hipótesis propuestas anteriormente. Esto va a permitir a las empresas evaluar esta utilidad y disponer de aquellos atributos que son valorados por el consumidor y le agrega valor en relación con las redes sociales del sector hotelero. El presente estudio se ha realizado a través de las páginas de Facebook y Twitter de los hoteles o cadena de hoteles de tres a cinco estrellas. Estas dos redes sociales han sido elegidas porque son las más utilizadas por las empresas del sector hotelero (Hmobile, 2015; Beezhotels, 2016). Para la construcción del cuestionario se ha llevado a cabo una búsqueda bibliográfica extensa sobre los ítems que son más adecuados para el estudio, los cuáles han sido tratados y aprobados por otros autores. A continuación, en la Tabla 2 se muestran las variables a analizar y los ítems a medir.

Los participantes se seleccionaron por método no probabilístico de sujetos voluntarios. La población para el presente estudio está formada por fans o seguidores de las páginas de Facebook o Twitter de hoteles. Para el desarrollo de esta investigación se solicitó mediante correo electrónico y teléfono colaboración a los hoteles de la provincia de Cádiz de tres o cinco estrellas que tuvieran presencia en Facebook o Twitter con al menos 300 seguidores, para que añadieran el enlace de la encuesta online en su página de red social. Finalmente, un total de 7 hoteles accedieron a incluir la encuesta.

La encuesta online al estar a disposición de las redes sociales de los hoteles y al ser dirigida a seguidores se aseguró que fuera respondida por aquellos usuarios que han estado presente en dicha página del hotel y han comprobado su contenido. Por lo tanto, se distribuyó a usuarios tanto hombres como mujeres, de al menos 18 años de edad. Por lo tanto, las condiciones para participar en el estudio son: tener una cuenta de Facebook o Twitter, y a su vez, seguir por Facebook o Twitter a alguna empresa del sector hotelero. Como la encuesta ha sido publicada por los propios hoteles, se facilita que estas condiciones se cumplan en todos aquellos que participen. En la Tabla 3 se especifica la ficha técnica de este estudio cuantitativo.

Tabla 2: Variables e ítems de la investigación cuantitativa.

\begin{tabular}{|c|c|c|}
\hline Variables & Items de medición & Referencia \\
\hline $\begin{array}{l}\text { Experiencia del usuario con } \\
\text { la página de social media } \\
\text { del hotel }\end{array}$ & $\begin{array}{l}\text { Nada interesante - muy interesante } \\
\text { Nada relevante - muy relevante } \\
\text { Nada emocionante - muy emocionante } \\
\text { Nada atractiva - muy atractiva } \\
\text { Nada agradable - muy agradable } \\
\text { Nada útil - muy útil } \\
\text { Nada entretenida - muy entretenida } \\
\text { Nada necesaria - muy necesaria } \\
\text { Nada beneficiosa - muy beneficiosa } \\
\text { Nada interactiva - muy interactiva }\end{array}$ & Leung et al. (2013) \\
\hline Beneficios funcionales & $\begin{array}{l}\text { Obtener información actualizada sobre el hotel } \\
\text { Obtener un contenido atractivo } \\
\text { Poder interactuar directamente con el hotel } \\
\text { Ver imágenes o videos del hotel }\end{array}$ & $\begin{array}{l}\text { De Vries y Carlson } \\
(2014) ; \text { Kang }(2011) \\
\text { Wang y Fesenmaier } \\
(2004)\end{array}$ \\
\hline $\begin{array}{l}\text { Beneficios socio- } \\
\text { psicológicos }\end{array}$ & $\begin{array}{l}\text { Poder compartir tus propias experiencias u opiniones } \\
\text { con otros miembros de la comunidad } \\
\text { Conocer las experiencias u opiniones de otros } \\
\text { miembros de la comunidad } \\
\text { Establecer y mantener relaciones con otros miembros } \\
\text { de la comunidad } \\
\text { Crear un sentido de afiliación o pertenencia con la } \\
\text { comunidad } \\
\text { Confiar más en el hotel }\end{array}$ & $\begin{array}{l}\text { Kang (2011); Wang y } \\
\text { Fesenmaier (2004) }\end{array}$ \\
\hline Beneficios hedónicos & $\begin{array}{l}\text { Buscar entretenimiento en la página de } \\
\text { Facebook/Twitter del hotel } \\
\text { Disfrutar en la página de Facebook/Twitter del hotel } \\
\text { Buscar diversión con otros miembros de la comunidad }\end{array}$ & $\begin{array}{l}\text { Kang (2011); Wang y } \\
\text { Fesenmaier (2004) }\end{array}$ \\
\hline Beneficios monetarios & $\begin{array}{l}\text { Obtener descuentos u ofertas especiales } \\
\text { Obtener mejores precios } \\
\text { Recibir cupones } \\
\text { Participar en concursos }\end{array}$ & $\begin{array}{l}\text { Gummerus et al. } \\
\text { (2012); Gwinner et al. } \\
(1998) ; \text { Lee et al. } \\
(2008)\end{array}$ \\
\hline
\end{tabular}


Tabla 3: Ficha técnica del estudio cuantitativo.

\begin{tabular}{|l|l|}
\hline Universo & $\begin{array}{l}\text { La población para el presente estudio está formada por los fans o seguidores de las } \\
\text { páginas de Facebook o Twitter de hoteles }=>3.000 .000 \text { (anexo IV) }\end{array}$ \\
\hline Muestra & 164 encuestas válidas \\
\hline Nivel de confianza & $\alpha=95,5 \%$ \\
\hline Error de muestreo & $7,8 \%$ (utilizando el supuesto de máxima indeterminación $\mathrm{p}=\mathrm{q}=50 \%)$ \\
\hline Tipo de muestreo & Por método no probabilístico de sujetos voluntarios. \\
\hline Trabajo de campo & Realizado en febrero-marzo de 2017 \\
\hline
\end{tabular}

\section{RESULTADOS}

Se han obtenido en total 164 respuestas al cuestionario, todos ellos seguidores de una página de Facebook o Twitter de un hotel o cadena de hoteles. Para el contraste de hipótesis se empleó la técnica de regresión. Se estimaron 3 modelos de regresión lineal simple para las hipótesis 1 a 3 y un modelo de regresión logística para la 4, ya que para la variable "beneficios monetarios" no sigue una distribución normal y por lo tanto no es apropiado usar una regresión lineal para analizar la relación de dependencia entre beneficios monetarios y experiencia en las redes sociales de los hoteles, pues no se cumplen los supuestos teóricos básicos de la regresión lineal. Por esta razón, se utiliza una regresión logística, que va a permitir modelar y analizar adecuadamente la relación entre las dos variables. Tal como explica Moore (2009) la regresión es un modelo matemático que permite predecir el valor de una variable dependiente $(Y)$ en función de los valores de la variable independiente $(X)$. Dicho de otra forma, los valores de $Y$ son función de los valores de $X$. El término lineal se refiere a que la predicción se restringe a variables entre las cuales existe una relación lineal. Esto quiere decir que la representación gráfica de la relación entre $X$ e $Y$ genera una línea recta o una aproximación de ésta. Según Hair et al. (2007), la regresión lineal es una técnica de dependencia puesto que analiza la relación entre una variable dependiente (o criterio) y una o varias variables independientes (o predictores). En lo que sigue se especifican los resultados alcanzados para cada una de las 4 relaciones entre las variables.

\section{Experiencia en las redes sociales y beneficios funcionales}

En la Tabla 4 se muestra el resultado de la regresión lineal entre estas variables. La variable independiente "experiencia" en las redes sociales de los hoteles es capaz de explicar el $31,8 \%$ de la variabilidad de los beneficios funcionales. Además, el gráfico de dispersión de beneficios funcionales y experiencia en las redes sociales muestra una nube de puntos algo dispersa $(r=0,564)$, pero con forma lineal y ausencia de otros patrones que indiquen no linealidad.

Tabla 4: Resumen del modelo ${ }^{\mathrm{b}}$ (beneficio funcional y experiencia)

\begin{tabular}{|c|c|c|c|c|}
\hline Modelo & $R$ & $R$ cuadrado & $R$ cuadrado corregida & Error típ. de la estimación \\
\hline 1 &, $564^{\text {a }}$ &, 318 &, 314 & 1,99895 \\
\hline
\end{tabular}

Se especifica que la variable independiente "experiencia" en las redes sociales tiene una contribución significativa en la explicación de la variabilidad de la variable dependiente "beneficios funcionales", tal como indica el estadístico $t$ de Student para el coeficiente de regresión ( $t=8,692, p<0,001)$. Se trata de una relación positiva, concretamente, el aumento de un punto en la escala de experiencia en las redes sociales de los hoteles produce un aumento de 0,158 puntos en la escala de beneficios funcionales (Tabla 5 y Figura 2). Los datos anteriores proporcionan evidencia estadística que permite corroborar la hipótesis 1 a un 99,9\% de confianza: "una mejor experiencia en la página de social media del hotel se asocia con un mayor beneficio funcional".

Tabla 5: Coeficientes ${ }^{a}$ (beneficio funcional y experiencia). a. Variable dependiente: Beneficios Funcionales

\begin{tabular}{|c|l|c|c|c|c|c|c|c|}
\hline \multirow{2}{*}{\multicolumn{2}{c|}{ Modelo }} & \multicolumn{2}{|c|}{$\begin{array}{c}\text { Coeficientes no } \\
\text { estandarizados }\end{array}$} & $\begin{array}{c}\text { Coeficientes } \\
\text { tipificados }\end{array}$ & \multirow{2}{*}{$t$} & \multirow{2}{*}{ Sig. } & $\begin{array}{c}\text { Intervalo de confianza de } \\
\text { 95,0\% para B }\end{array}$ \\
\cline { 3 - 9 } & $B$ & Error típ. & Beta & & $\begin{array}{c}\text { Límite } \\
\text { inferior }\end{array}$ & $\begin{array}{c}\text { Límite } \\
\text { superior }\end{array}$ \\
\hline \multirow{2}{*}{1} & 16,240 &, 994 & & 16,345 &, 000 & 14,278 & 18,202 \\
\hline & $\begin{array}{l}\text { (Constante) } \\
\text { Experiencia en las } \\
\text { redes sociales de los } \\
\text { hoteles }\end{array}$ &, 158 &, 018 &, 564 & 8,692 &, 000 &, 122 &, 194 \\
\hline
\end{tabular}




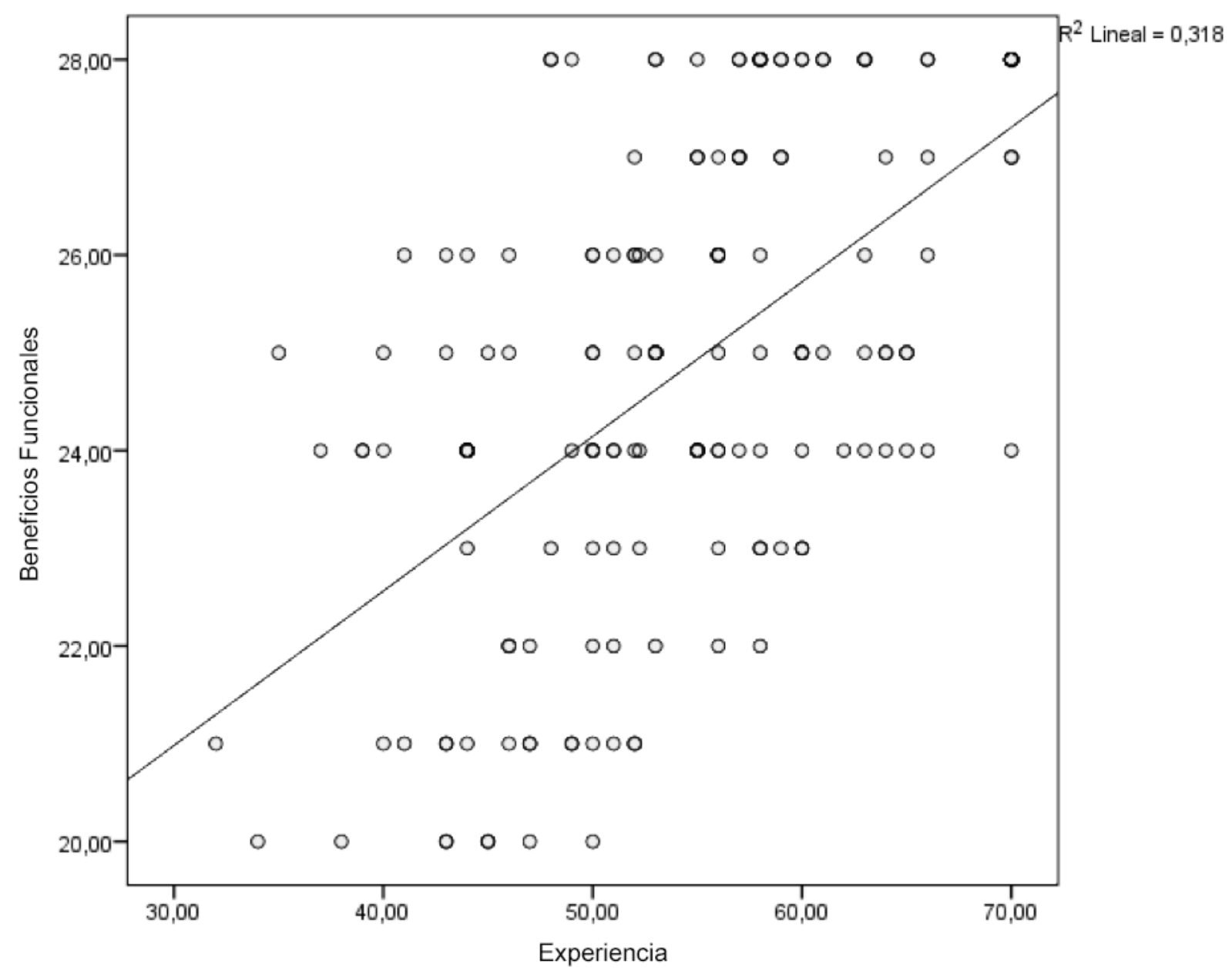

Fig. 2: Relación entre beneficio funcional y experiencia.

\section{Experiencia en las redes sociales y beneficios socio-psicológicos}

En la Tabla 6 se muestra el resultado de la regresión lineal entre estas variables. La variable independiente "experiencia" es capaz de explicar el $28,5 \%$ de la variabilidad de los beneficios socio-psicológicos. Además, el gráfico de dispersión de beneficios funcionales y experiencia en las redes sociales de hoteles muestra una nube de puntos algo dispersa $(r=0,534)$, pero con una forma claramente lineal y ausencia de otros patrones que indiquen no linealidad.

Tabla 6: Resumen del modelob (beneficio socio-psicológico y experiencia)

\begin{tabular}{|c|c|c|c|c|}
\hline Modelo & $\mathrm{R}$ & $\mathrm{R}$ cuadrado & $\mathrm{R}$ cuadrado corregida & Error típ. de la estimación \\
\hline 1 &, $534^{\mathrm{a}}$ &, 285 &, 280 & 3,88980 \\
\hline
\end{tabular}

a. Variables predictoras: (Constante), Experiencia en las redes sociales de los hoteles

b. Variable dependiente: Beneficios socio-psicológicos

Se determina que la variable independiente "experiencia" en las redes sociales de los hoteles tiene una contribución significativa en la explicación de la variabilidad de la variable dependiente "beneficios funcionales", tal como indica el estadístico t de Student para el coeficiente de regresión $(t=7,933, p<0,001)$. Se trata de una relación positiva, concretamente, el aumento de un punto en la escala de experiencia produce un aumento de 0,287 puntos en la escala de beneficios socio-psicológicos (Tabla 7 y Figura 3). Los datos anteriores proporcionan evidencia estadística que permite corroborar la hipótesis 2 a un 99,9\% de confianza: "una mejor experiencia en la página de social media del hotel se asocia con un mayor beneficio sociopsicológico" 
Tabla 7: Coeficientesa (beneficio socio-psicológico y experiencia). a. Variable dependiente: Beneficios socio-psicológicos

\begin{tabular}{|c|c|c|c|c|c|c|c|c|}
\hline & \multirow{2}{*}{ Modelo } & \multicolumn{2}{|c|}{$\begin{array}{l}\text { Coeficientes no } \\
\text { estandarizados }\end{array}$} & \multirow{2}{*}{$\begin{array}{c}\begin{array}{c}\text { Coeficientes } \\
\text { tipificados }\end{array} \\
\text { Beta }\end{array}$} & \multirow{2}{*}{$\mathrm{t}$} & \multirow{2}{*}{ Sig. } & \multicolumn{2}{|c|}{$\begin{array}{c}\text { Intervalo de confianza de } \\
95,0 \% \text { para B }\end{array}$} \\
\hline & & B & Error típ. & & & & $\begin{array}{l}\text { Límite } \\
\text { inferior }\end{array}$ & $\begin{array}{l}\text { Límite } \\
\text { superior }\end{array}$ \\
\hline & (Constante) & 12,351 & 1,982 & & 6,232 & ,000 & 8,436 & 16,265 \\
\hline 1 & $\begin{array}{l}\text { Experiencia en } \\
\text { las redes } \\
\text { sociales de los } \\
\text { hoteles }\end{array}$ & ,287 & ,036 &, 534 & 7,933 & ,000 & ,216 & ,358 \\
\hline
\end{tabular}

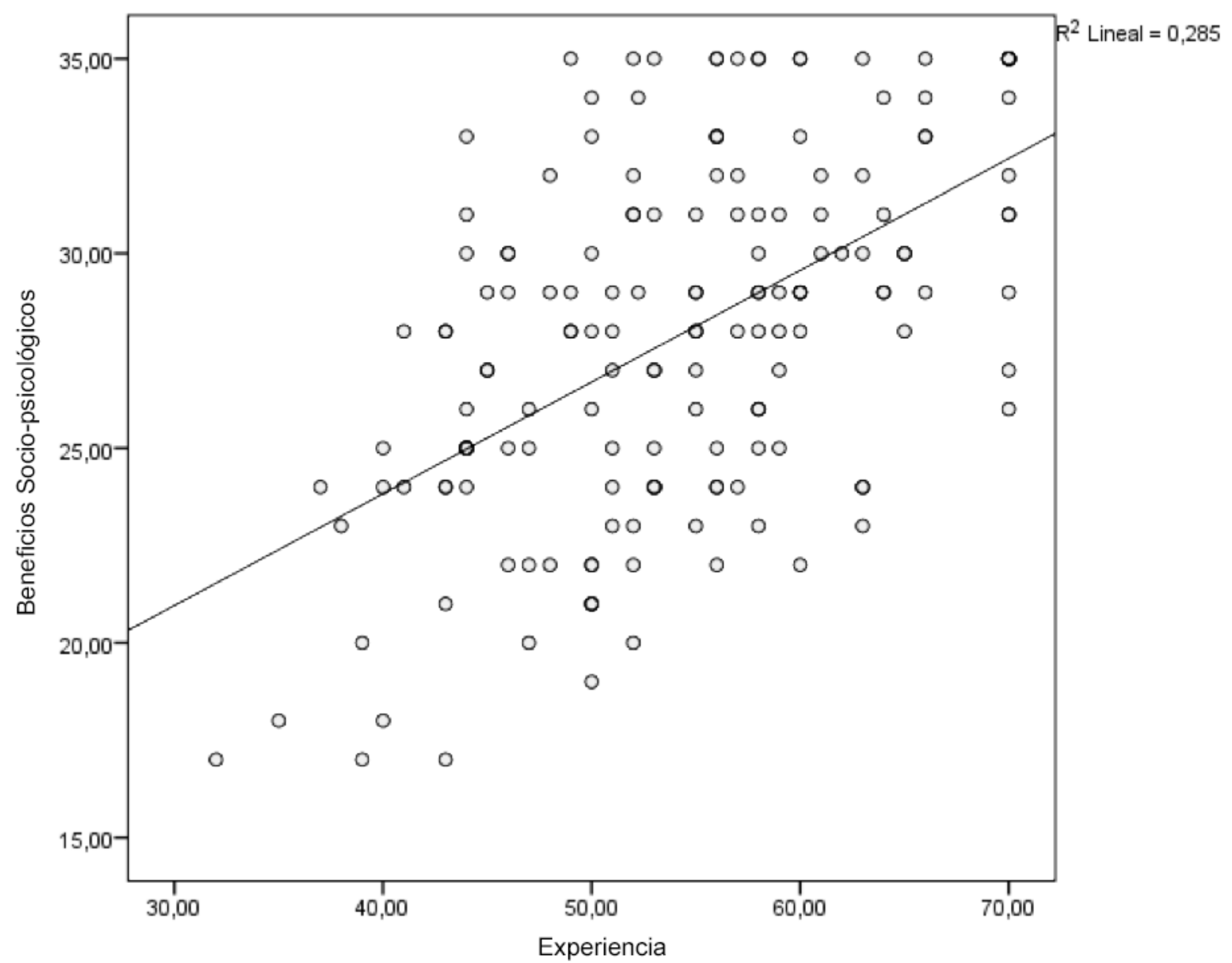

Fig. 3: Relación entre beneficio socio-psicológico y experiencia.

Experiencia en las redes sociales y beneficios hedónicos

En la Tabla 8 se muestra el resultado de la regresión lineal entre estas variables. La variable independiente "experiencia" es capaz de explicar el $32 \%$ de la variabilidad de los beneficios hedónicos. Además, el diagrama de dispersión de beneficios hedónicos y experiencia muestra una nube de puntos menos dispersa que en los modelos anteriores $(r=0,565)$, y con una forma claramente lineal y total ausencia de otros patrones que indiquen no linealidad.

Tabla 8: Resumen del modelob (beneficio hedónico y experiencia)

\begin{tabular}{|c|c|c|c|c|}
\hline Modelo & $R$ & $R$ cuadrado & $R$ cuadrado corregida & Error típ. de la estimación \\
\hline 1 &, $565^{\mathrm{a}}$ &, 320 &, 315 & 2,96539 \\
\hline
\end{tabular}


Se especifica que la variable independiente "experiencia" en las redes sociales de los hoteles tiene una contribución significativa en la explicación de la variabilidad de la variable dependiente "beneficios funcionales", tal como indica el estadístico t de Student para el coeficiente de regresión $(t=8,535, p<0,001)$. Se trata de una relación positiva, concretamente, el aumento de un punto en la escala de experiencia produce un aumento de 0,241 puntos en la escala de beneficios hedónicos (Tabla 9 y Figura 4). Los datos anteriores proporcionan evidencia estadística que permite corroborar la hipótesis 3 a un 99,9\% de confianza: "una mejor experiencia en la página de social media del hotel se asocia con un mayor beneficio hedónico".

Tabla 9: Coeficientesa (beneficio hedónico y experiencia). a. Variable dependiente: Beneficios Hedónicos

\begin{tabular}{|c|c|c|c|c|c|c|}
\hline & \multirow{2}{*}{ Modelo } & \multicolumn{2}{|c|}{ Coeficientes no estandarizados } & \multirow{2}{*}{$\begin{array}{c}\text { Coeficientes } \\
\text { tipificados } \\
\text { Beta }\end{array}$} & \multirow[t]{2}{*}{$t$} & \multirow{2}{*}{ Sig. } \\
\hline & & $B$ & Error típ. & & & \\
\hline \multirow[b]{2}{*}{1} & (Constante) & 1,008 & 1,552 & &, 650 &, 517 \\
\hline & $\begin{array}{l}\text { Experiencia en } \\
\text { las redes } \\
\text { sociales de los } \\
\text { hoteles }\end{array}$ & ,241 & ,028 &, 565 & 8,535 &, 000 \\
\hline
\end{tabular}

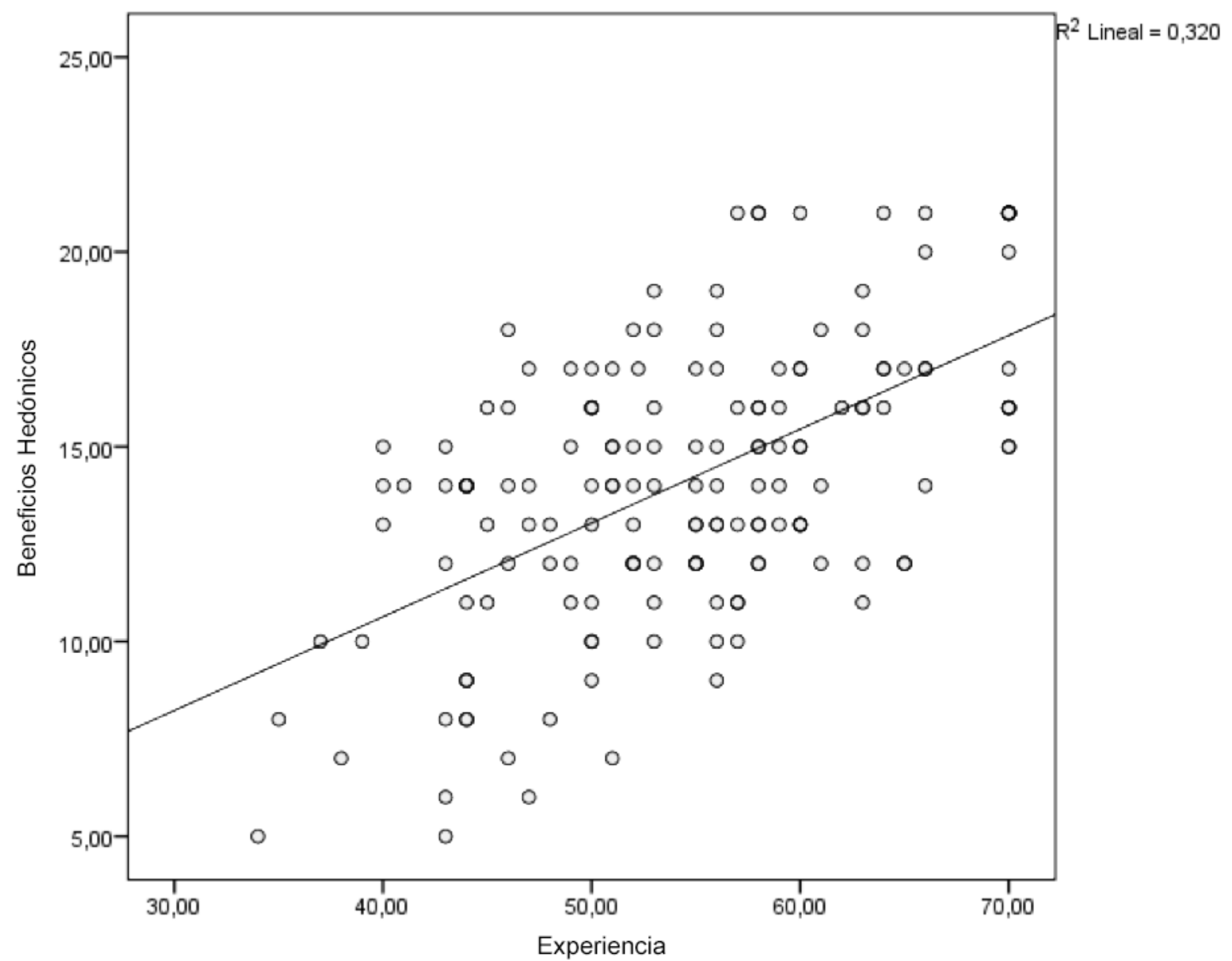

Fig. 4: Relación entre beneficio hedónico y experiencia.

\section{Experiencia en las redes sociales y beneficios monetarios}

En esta relación se confirma la anormalidad de la variable "beneficios monetarios" que afecta a la distribución de los residuos de la regresión. Por lo tanto, para contrastar esta relación se utiliza la regresión logística binaria. La regresión logística permite predecir la probabilidad de que ocurra un evento frente a la posibilidad de que no ocurra. En el caso de esta regresión, se identifican dos grupos, los encuestados que valoran con puntuaciones muy altas (27 o 28 puntos) y el resto de encuestados, que más o menos constituyen una $50 \%$ de la muestra. El evento queda definido como la probabilidad de que un encuestado tenga una alta puntuación (27 o más puntos) en la escala de beneficios monetarios, que se codifica como 1. Frente a ésta se calcula la probabilidad de que no ocurra dicho evento, es decir que el encuestado no tenga 27 o más puntos, o dicho 
de otra forma que tenga una puntuación media o baja (26 o menos puntos), que se codifica como 0 . Si la variable independiente "experiencia" en redes sociales de los hoteles influye significativamente en la variable "beneficios monetarios" su impacto se verá reflejado en estas probabilidades.

Una vez realizada se verifica en la Tabla 10 el impacto de la variable independiente "experiencia" en las redes sociales de los hoteles sobre la variable dependiente "beneficios monetarios", el cual se ve reflejado en el exponencial del coeficiente beta (Exp. B). Un encuestado con puntuación alta en experiencia en las redes sociales de los hoteles $(x=1)$ tiene 2,7 veces más probabilidades de otorgar una alta importancia a los beneficios monetarios $(\mathrm{y}=1)$ que otro entrevistado que tenga baja experiencia en las redes sociales de los hoteles $(x=0)$. Este aumento de la probabilidad es estadísticamente significativo a un $99 \%$ de confianza $(p=0,002)$, por lo cual se cumple la hipótesis 4: "una mejor experiencia en la página de social media del hotel se asocia con un mayor beneficio monetario".

Tabla 10: Variables en la ecuación. a. Variable(s) introducida(s) en el paso 1: Experiencia en las redes sociales de los hoteles

\begin{tabular}{|c|c|c|c|c|c|c|c|c|c|}
\hline & & \multirow{2}{*}{$B$} & \multirow{2}{*}{ E.T. } & \multirow{2}{*}{ Wald } & \multirow{2}{*}{$g l$} & \multirow{2}{*}{ Sig. } & \multirow{2}{*}{$\operatorname{Exp}(B)$} & \multicolumn{2}{|c|}{ I.C. $95 \%$ para $E X P(B)$} \\
\hline & & & & & & & & Inferior & Superior \\
\hline \multirow[t]{2}{*}{ Paso $1^{a}$} & $\begin{array}{c}\text { Experiencia en las } \\
\text { redes sociales de } \\
\text { los hoteles } \\
(1=\text { alta })\end{array}$ & ,996 & ,322 & 9,562 & 1 & ,002 & 2,708 & 1,440 & 5,093 \\
\hline & Constante &,- 550 & ,229 & 5,756 & 1 & ,016 &, 577 & & \\
\hline
\end{tabular}

\section{Contraste de las hipótesis}

En este apartado se relacionan a modo de resumen en la Tabla 11 los resultados derivados del contraste de las hipótesis planteadas en el estudio, ya comentadas en el punto anterior.

Tabla 11: Contraste de hipótesis en los modelos de regresión lineal simple y regresión logística (hipótesis 1, 2, 3 y 4).

\begin{tabular}{|c|c|c|c|c|c|c|c|c|}
\hline \multicolumn{7}{|c|}{ Modelos de regresión lineal simple } \\
\hline Hip. & VD & VI & $R^{2}$ & $B$ & $B e t a$ & $t$ & $p$ & Decisión \\
\hline 1 & BFUN & EXP & 0,318 & 0,158 & 0,564 & 8,692 & $<0,001$ & Contrastada \\
\hline 2 & BSOC & EXP & 0,285 & 0,287 & 0,534 & 7,933 & $<0,001$ & Contrastada \\
\hline 3 & BHED & EXP & 0,320 & 0,241 & 0,565 & 8,535 & $<0,001$ & Contrastada \\
\hline \multicolumn{7}{|c|}{ Modelo de regresión logística binaria } \\
\hline Hip. & VD & VI & $R 2$ Cox-Snell & $R 2$ Nagelkerke & Exp(B) & $p$ & Decisión \\
\hline 4 & BMON & EXP & 0,058 & 0,078 & 2,708 & 0,002 & Contrastada \\
\hline
\end{tabular}

\section{DISCUSIÓN FINAL}

Respecto a la relación entre la experiencia del usuario en la página del hotel en las redes sociales y los beneficios percibidos, se ha contrastado empíricamente que es positiva en los cuatro tipos de beneficios que se han estudiado: funcional, socio-psicológico, hedónico y monetario. De esta manera, se puede determinar que mediante una experiencia positiva y satisfactoria de los usuarios en la red social estos percibirán mayor beneficio funcional, entendido este como la facilidad en la obtención relevante por parte del usuario. También la percibirán un mayor beneficio socio-psicológico, en el que los usuarios obtienen una mayor conexión, vinculación y confianza con otros usuarios en la red social. Además, los usuarios obtienen un mayor beneficio hedónico en la relación con la experiencia, referido este como la obtención de entretenimiento y disfrute a través de la red social. Por último, una mejor experiencia en la red social del hotel también va a proporcionar un incremento del beneficio económico, en el que los usuarios buscan un ahorro o descuento en su intervención en la red social. El estudio pone de manifiesto la obtención de beneficios por parte de los usuarios en su intervención en las redes sociales, así como la de un mayor grado de compromiso. De esta manera, se resalta la importancia de ofrecer por parte de los hoteles un contenido atractivo y orientado a los usuarios a través de las redes sociales. 


\section{CONCLUSIONES}

De acuerdo al trabajo presentado y a los resultados obtenidos, se pueden plantear las siguientes conclusiones principales:

1.- Los usuarios van a buscar un provecho o utilidad en el tiempo que pasen en contacto con las redes sociales de los hoteles y van a esperar múltiples beneficios en su intervención. En primer lugar, los profesionales de la hostelería deben proporcionar un adecuado servicio funcional mediante una información y contenido atractivo y actualizado y el uso eficiente de material audiovisual. Los usuarios también pretenden participar en la co-creación de contenido especificando sus opiniones y experiencias en las redes, tomando en consideración la de los demás usuarios y esperando que estas sean tenidas en cuenta por el hotel. De esta manera, pretende obtener interacciones sociales, tanto con los demás usuarios como con el propio hotel, dando relevancia al beneficio socio-psicológico. Los usuarios no solo quieren ver o comprobar una determinada información o contenido, sino quieren divertirse y entretenerse mientras navegan en las redes sociales y buscan información, predominando así una experiencia tanto informativa como placentera. Por último, los costes se establecen como una preocupación esencial en los usuarios. Estos demandan descuentos y ofertas especiales, promoviendo a los hoteles utilizar promociones monetarias para estimular la compra en el corto plazo.

2.- Los resultados del estudio sugieren específicamente que la experiencia de los usuarios en las redes sociales de los hoteles influye en la obtención por parte de estos de beneficios funcionales, socio-psicológicos, hedónicos y monetarios. Esto indica que los hoteles deben centrarse en crear una experiencia positiva y satisfactoria para los usuarios en las redes sociales. Ya no basta solo con estar presente en las diferentes redes sociales, los gerentes de los hoteles deben crear y gestionar páginas de redes sociales útiles, valiosas y centradas en el consumidor.

\section{REFERENCIAS}

Arévalo-Avecillas, D.X., C.P. Padilla-Lozano, M. A. Bustamante-Ubilla y C. L. Vidal-Silva, Contrastación de la Paradoja de la Productividad por el uso de las Tecnologías de Información: el Caso Ecuatoriano, Información Tecnológica, 28(1), 171$178(2017)$

Barrera, G. A., Relación de Capacitación con Adopción de Internet y E-Commerce: Diferencias entre Microemprendedores de Chile, Información Tecnológica, 28(6), 61-70 (2017)

Brendzel-Skowera, K. y K. Lukasik, Polish Universities in Social Media, Valahian Journal of Economic Studies, 7(4), 29 (2016)

Bruhn, M., S. Schnebelen y D. Schäfer, Antecedents and consequences of the quality of e-customer-to-customer interactions in B2B brand communities, Industrial Marketing Management, 43(1), 164-176 (2014)

Butler, B.S., Membership size, communication activity, and sustainability: A resource-based model of online social structures, Information Systems Research, 12(4), 346-362 (2001)

Chen, R., Member use of social networking sites-an empirical examination, Decision Support Systems, 54(3), 1219-1227 (2013)

Dans, E., Seguimiento y evaluación: mitos y leyendas online en la nueva era digital 2.0, Corporate excellence (2011)

De Vries, N.J. y J. Carlson, Examining the drivers and brand performance implications of customer engagement with brands in the social media environment, Journal of Brand Management, 21(6), 495-515 (2014)

Dholakia, U.M., R.P. Bagozzi y L. K. Pearo, A social influence model of consumer participation in network-and small-groupbased virtual communities, International journal of research in marketing, 21(3), 241-263 (2004)

Furlong, M. S., An electronic community for older adults: The SeniorNet network, Journal of Communication, 39(3), 145$153(1989)$

$\mathrm{Gu}$, B. y S. Jarvenpaa, Online discussion boards for technical support: the effect of token recognition on customer contributions, ICIS 2003 Proceedings, 10 (2003)

Gwinner, K. P., D. D. Gremler y M.J. Bitner, Relational benefits in services industries: the customer's perspective, Journal of the academy of marketing science, 26(2), 101-114 (1998)

Jiménez-Pitre, I. A., R. J. Martelo y J. D. Jaimes, Escuela de Gobierno basada en TIC: Determinante para la Accesibilidad e Integralidad del Empoderamiento Digital, Información Tecnológica, 28(5), 75-86 (2017)

Kang, I., K.C. Lee, S. Lee y J. Choi, Investigation of online community voluntary behavior using cognitive map, Computers in Human Behavior, 23(1), 111-126 (2007)

Kang, J., Social media marketing in the hospitality industry: the role of benefits in increasing brand community participation and the impact of participation on consumer trust and commitment toward hotel and restaurant brands, Dissertations \& Theses Global (2011) 
Lee, Y.K., W.K. Ahn y K. Kim, A study on the moderating role of alternative attractiveness in the relationship between relational benefits and customer loyalty, International Journal of hospitality \& tourism administration, 9(1), 52-70 (2008)

Leung, X. Y., B. Bai y K. A. Stahura, The marketing effectiveness of social media in the hotel industry: A comparison of Facebook and Twitter, Journal of Hospitality \& Tourism Research, 39(2), 147-169 (2015)

Madupu, V., Online brand community participation: antecedents and consequences, Dissertations \& Theses Global (2006)

Martelo, R. J., I. A. Jiménez y J. D. Jaimes, Accesibilidad e Integración Digital: Elementos Clave para un Programa de Formación de Empresarios en Empoderamiento Digital, Información Tecnológica, 28(6), 81-94 (2017)

Martí, J., Hypermarcas y marketing de compromisos, MK: marketing + ventas, (237), 5055 (2008)

Martínez, S. M, J. J. Bernal García y J. P. Mellinas, Análisis del nivel de presencia de los establecimientos hoteleros de la Región de Murcia en la Web 2.0, Cuadernos de Turismo, (31), 245-261 (2013)

Moreno, M., El gran libro del Community Manager, Gestión, Barcelona, España (2014)

Morgan, R. M. y S. D. Hunt, The commitment-trust theory of relationship marketing, The journal of marketing, 20-38 (1994)

Naumann, K. y J. Lay-Hwa Bowden, Exploring the process of customer engagement, self-brand connections and loyalty, Problems and perspectives in management (2015)

Potgieter, L.M. y R. Naidoo, Factors explaining user loyalty in a social media-based brand community, South African Journal of Information Management, 19(1), 1-9 (2017)

Rosenthal, B. y E. P. Brito, How virtual brand community traces may increase fan engagement in brand pages, Business Horizons, 60(3), 375-384 (2017)

Sigala, M., Web 2.0 in the tourism industry: A new tourism generation and new ebusiness models (2007)

Sirakaya, E. y A. Woodside, Building and testing theories of decision making by travelers, Tourism Management, 26(6), 815-832 (2005)

Song, S. y M. Yoo, The role of social media during the pre-purchasing stage, Journal of Hospitality and Tourism Technology, 7(1), 84-99 (2016)

Van der Lans, R., G. Van Bruggen, J. Eliashberg, J. y B. Wierenga, Predicting and Optimizing the spread of electronic word of mouth, GFK marketing intelligence Review, 4(1), 32-41 (2012)

Vila, T.D. y N.A. Vila, El fenómeno 2.0 en el sector turístico, El caso de Madrid 2.0, PASOS, Revista de Turismo y Patrimonio Cultural, 10(3), 225-237 (2012)

Vivek, S., S. Beatty y R. Morgan, Customer engagement: Exploring customer relationships beyond purchase, Journal of Marketing Theory and Practice, 20(2), 122-146 (2012)

Wang, Y. y D. R. Fesenmaier, Towards understanding members' general participation in and active contribution to an online travel community, Tourism management, 25(6), 709-722 (2004)

Wang, Y., Q. Yu y D.R. Fesenmaier, Defining the virtual tourist community: implications for tourism marketing, Tourism management, 23(4), 407-417 (2002)

Zimmerman, J. y D. Ng, Social media marketing all-in-one for dummies John Wiley \& Sons, New Jersey, USA (2015) 